\title{
Thickness-Dependent Defect Evolution in GaAs0.92Sbo.08/GaAs Heterostructures
}

\author{
Abhinandan Gangopadhyay ${ }^{1}$, Aymeric Maros $^{2}$, Nikolai Faleev ${ }^{2}$ and David J. Smith ${ }^{3}$ \\ 1. School for Engineering of Matter, Transport and Energy, Arizona State University, Tempe, AZ \\ 2. School of Electrical, Computer and Energy Engineering, Arizona State University, Tempe, AZ \\ 3. Department of Physics, Arizona State University, Tempe, AZ
}

$\mathrm{GaAs}_{1-\mathrm{x}} \mathrm{Sb}_{\mathrm{x}} / \mathrm{GaAs}$ heterostructures are promising candidates for intermediate-band solar cells (IBSCs). Ternary compounds such as $\mathrm{GaAs}_{1-\mathrm{x}} \mathrm{Sb}_{\mathrm{x}}$ facilitate band-gap tuning by introducing an intermediate band between valence and conduction bands. The efficiency of IBSCs can ideally be as high as $63.7 \%$ owing to additional photon absorptions related to electron transitions between valence band to intermediate band and intermediate band to conduction band [1]. A major obstacle to achieving high efficiency is the presence of crystallographic defects which act as non-radiative recombination centers. Understanding defect evolution during crystal growth and subsequently minimizing defects by adjusting growth conditions should improve device performance. Defect-free pseudomorphic growth of mismatched materials enabled by molecular beam epitaxy (MBE) is possible up to some critical thickness as the lattice mismatched epilayer is elastically strained to match the substrate lattice. Beyond the critical thickness the accumulated elastic strain is partially relieved by introduction of misfit dislocations. The nature, distribution and density of heteroepitaxial defects are the result of complex inter-related factors such as growth temperature, lattice mismatch, quality of substrate and thickness of epilayer [2]. This study describes the characterization of defect evolution as a function of epilayer thickness in GaAs 1 ${ }_{\mathrm{x}} \mathrm{Sb}_{\mathrm{x}} / \mathrm{GaAs}$ heterostructures grown by MBE using GaAs (001) substrates. The Sb content of the epilayers was measured to be $\sim 8 \%$ using high-resolution $\mathrm{x}$-ray diffraction. Cross-sectional TEM samples were prepared using conventional polishing, dimpling and argon ion milling. The milling was carried out at $2.7 \mathrm{keV}$ with samples held at liquid nitrogen temperature in order to minimize any milling damage. A Philips-FEI CM-200 microscope operated at $200 \mathrm{keV}$ was used for imaging. Results are reported for $\mathrm{GaAs}_{0.92} \mathrm{Sb}_{0.08} / \mathrm{GaAs}$ heterostructures with thicknesses in the range of 50 to $4000 \mathrm{~nm}$.

Figure 1(a) shows a schematic cross-sectional view of the investigated $\mathrm{GaAs}_{0.92} \mathrm{Sb}_{0.08} / \mathrm{GaAs}$ heterostructures, while Fig. 1(b) illustrates that the heterostructure with a film thickness of $50 \mathrm{~nm}$ showed no defects at the interface, which is as expected since this film thickness is considerably less than the calculated critical thickness of $93 \mathrm{~nm}$ given the $0.58 \%$ lattice mismatch between the two materials. Figure 2(a) shows the presence of dislocations at the interface in the heterostructure with 100nm-thick $\mathrm{GaAs}_{0.92} \mathrm{Sb}_{0.08}$ layer. The contrast modulations observable in Fig. 2(b) for the same sample are indicative of slight compositional inhomogeneities in the film during growth, also observed by photoluminescence [3]. Substantial interfacial defects were present in thicker $\mathrm{GaAs}_{0.92} \mathrm{Sb}_{0.08}$ layers. For example, the interfaces of 500-nm-thick and 4- $\mu$ m-thick GaAs $0.92 \mathrm{Sb}_{0.08}$ layers with the GaAs buffer layer show the presence of a high density of misfit dislocations, as evident from Fig. 3(a) and (b). Further observations of materials with intermediate thicknesses are ongoing to gain more detailed information about the mechanisms for stress relaxation via interfacial dislocations and threading defects [4].

[1] A. Luque and A. Martí, Phys. Rev. Lett. 78 (1997), p. 5014.

[2] K. H. Chang, P. K. Bhattacharya and R. Gibala, J. Appl. Phys. 66 (1989), p. 2993.

[3] A. Maros et al, J. Appl. Phys. 120 (2016), p. 183104.

[4] This work was supported by the National Science Foundation and the Department of Energy under NSF CA No. EEC-1041895. The authors acknowledge the use of facilities within the John M. Cowley Center for High Resolution Electron Microscopy at Arizona State University. 


\begin{tabular}{|c|}
\hline $\begin{array}{l}\text { (a) } \\
\text { GaAs capping layer }(50 \mathrm{~nm})\end{array}$ \\
\hline $\mathrm{GaAs}_{0.92} \mathrm{Sb}_{0.08}$ epilayer \\
\hline GaAs buffer layer \\
\hline GaAs (001) substrate \\
\hline
\end{tabular}

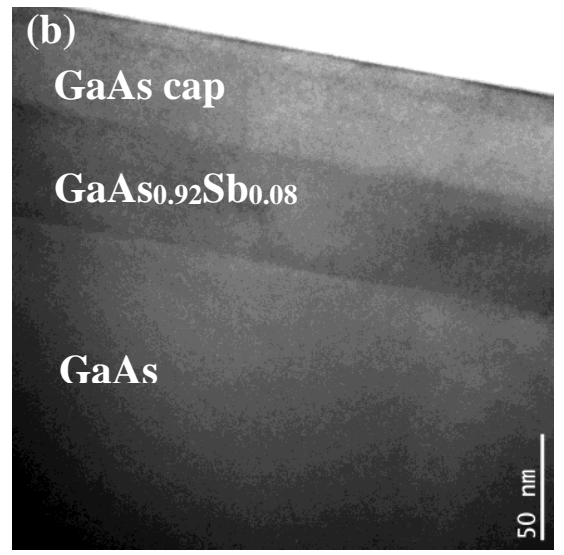

Figure 1. (a) Schematic cross-sectional view of $\mathrm{GaAs}_{0.92} \mathrm{Sb}_{0.08} / \mathrm{GaAs}$ heterostructure, (b) low magnification TEM image of the heterostructure with 50-nm-thick epilayer.
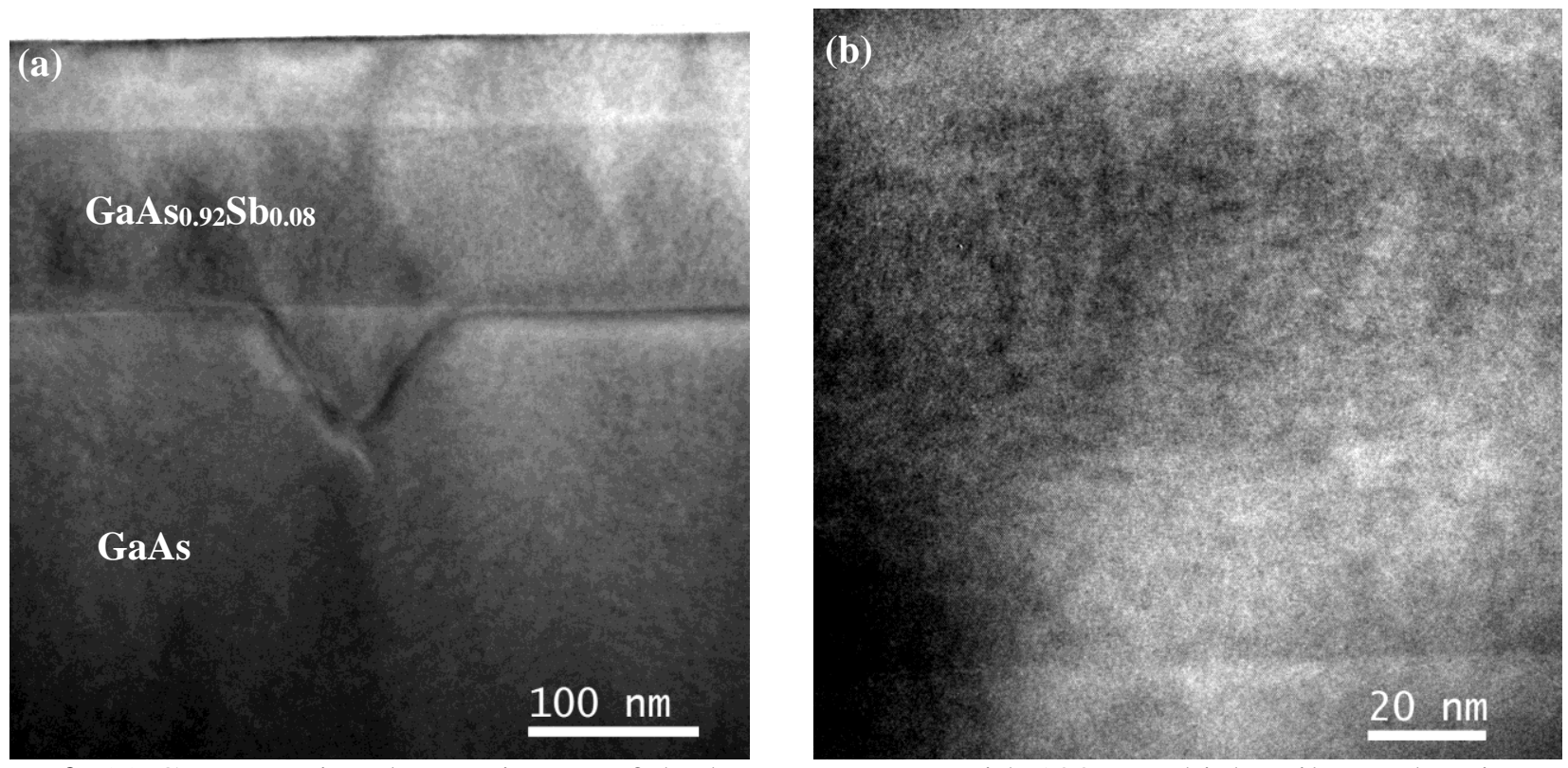

Figure 2. (a) Cross-sectional TEM image of the heterostructure with 100-nm-thick epilayer showing presence of interfacial defect, (b) Contrast modulation across the epilayer in the same heterostructure indicates slight compositional inhomogeneity.

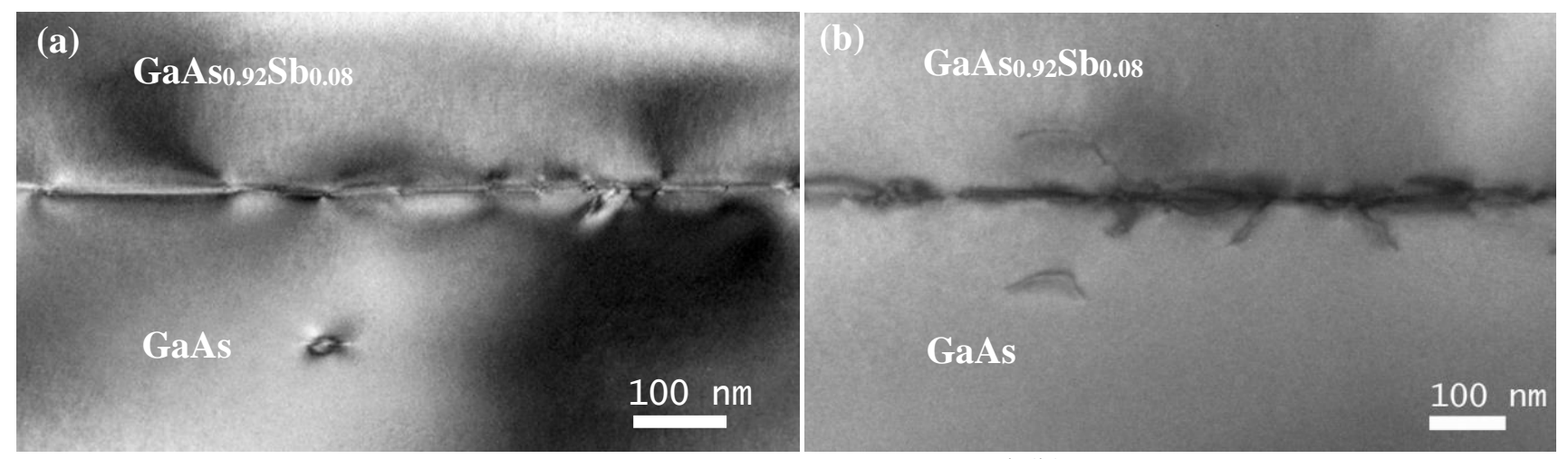

Figure 3. TEM images of the heterostructures with (a) $500 \mathrm{~nm}$, and (b) $4 \mu \mathrm{m}$, epilayers showing misfit dislocations at $\mathrm{GaAs}_{0.92} \mathrm{Sb}_{0.08} / \mathrm{GaAs}$ interface. 